\title{
Synergistic effect of smoking and blood pressure on augmentation index in men, but not in women
}

\author{
Hirofumi Tomiyama ${ }^{1}$, Mutsuo Yamazaki ${ }^{2}$, Yasuhiro Sagawa ${ }^{2}$, Kunihiko Teraoka ${ }^{3}$, Tsunemichi Shirota ${ }^{3}$, \\ Yoshinori Miyawaki ${ }^{2}$ and Akira Yamashina ${ }^{1}$
}

This study was conducted in healthy Japanese subjects to examine the effects of age and gender on the relationship between the risk factors for cardiovascular disease (CVD) and augmentation index (AI), and the effects of clusters of those risk factors on Al. Radial arterial pressure wave analysis was used to obtain Al in 3675 men and 2919 women. Al was found to be higher in women than in men, and age-related increase in Al showed an attenuated curve in subjects aged $\geqslant 50$ years. A step-wise multivariate linear regression analysis showed that mean blood pressure and smoking are independent significant variables related to $\mathrm{Al}$ in men regardless of age, and in women aged $<50$ years, but not in women aged $\geqslant 50$ years. A general linear model univariate linear regression analysis showed that mean blood pressure and smoking had a significant interaction for their relation with Al in men, but not in women. In conclusion, among the risk factors for CVD, smoking and blood pressure were found to be independent factors related to increase in Al. Although age-related attenuation of increase in Al was confirmed in Japanese subjects, these risk factors may act to increase Al even in elderly subjects, at least in part. However, the effects of these factors on Al may differ based on gender, and these factors may act synergistically to increase Al in men. On the contrary, these factors may act independently in young women to increase Al without interaction, whereas only the blood pressure seems to increase $\mathrm{Al}$ in elderly women.

Hypertension Research (2009) 32, 122-126; doi:10.1038/hr.2008.20; published online 23 January 2009

Keywords: age; augmentation index; gender; risk factors

\section{INTRODUCTION}

Accumulating evidence suggest that increased arterial stiffness is an independent risk for cardiovascular disease (CVD) ${ }^{1-3}$ The augmentation index (AI) is a marker related to systemic arterial stiffness, and some studies reported that increased AI or central blood pressure estimated by AI predicts future cardiovascular events. ${ }^{4-6}$ Some studies reported that CVD risk factors affect AI. ${ }^{711}$ The age-related increase in AI shows an attenuated curve in subjects aged $>50$ years, and AI is thought to be less sensitive in reflecting arterial stiffness in elderly subjects. ${ }^{12}$ Although AI increases predominantly in women, the Second Australian National Blood Pressure Study (ANBP 2 study) showed that it cannot be used as a marker for predicting future CVD events in elderly women with hypertension. ${ }^{13}$ Matsui et al. ${ }^{14}$ examined the effects of age and gender on AI in subjects with hypertension under anti-hypertensive medication. However, their study could not avoid the influence of anti-hypertensive medication on the results. Thus, the effects of both age and gender on the relationship between the risk factors for CVD and $\mathrm{AI}$ in healthy individuals have not been fully clarified. Furthermore, although it is noted that a cluster of those risk factors additively or synergistically augments the progression of atherosclerosis, ${ }^{15}$ the effect of the cluster of the risk factors on $\mathrm{AI}$ is also unclear.

The present cross-sectional study was conducted in a large number of healthy Japanese subjects (i.e., subjects with no known history of CVD and no medication for CVD or for its risk factors) to clarify the following issues: (1) the effect of both age and gender on the relationship between risk factors for CVD and AI, and (2) the effects of the cluster of those risk factors on AI.

\section{METHODS}

Study cohort

From the beginning of June 2006 to the end of November 2007, subjects who underwent health-screening examination at the Health Care Center of Tokyo Medical University were invited to participate in a cross-sectional study to examine the clinical significance of radial AI as a marker of CVD risk in Japanese subjects. In this study protocol, in addition to the routine health checkup including evaluation of the cardiovascular risk factors, pulmonary function test and measurement of the radial AI were conducted. Written informed consent was obtained from all participants prior to these measurements. The study was conducted with the approval of the Ethical Guidelines Committee of Tokyo Medical University.

\footnotetext{
1Second Department of Internal Medicine, Tokyo Medical University, Tokyo, Japan; ${ }^{2}$ Omron Healthcare Corporation, Kyoto, Japan and ${ }^{3}$ Center for Health Surveillance and Preventive Medicine, Tokyo Medical University, Tokyo, Japan

Correspondence: Professor H Tomiyama, Second Department of Internal Medicine, Tokyo Medical University, 6-7-1 Nishi-Shinjuku, Tokyo 160-0023, Japan.

E-mail: tomiyama@tokyo-med.ac.jp

Received 15 May 2008; revised 2 November 2008; accepted 28 November 2008; published online 23 January 2009
} 


\section{Measurements}

Augmentation index. The measurements of blood pressure and radial AI were conducted after the subjects had rested for $5 \mathrm{~min}$ in a sitting position, in an air-conditioned room $\left(24-26^{\circ} \mathrm{C}\right)$ earmarked exclusively for this purpose. The blood pressure was determined in the right upper arm using an oscillometric method (HEM-907; Omron Healthcare Co., Ltd, Kyoto, Japan). Then, the mean blood pressure was calculated as follows: the average diastolic blood pressure+(the average systolic blood pressure-the average diastolic blood pressure)/3. Immediately after this measurement, the left radial arterial waveform was obtained using an arterial applanation tonometry probe incorporating an array of 40 micropiezoresistive transducers (HEM-9010AI; Omron Healthcare Co., Ltd) ${ }^{16,17}$ The HEM-9010AI device is programmed to automatically determine the pressure against the radial artery to yield the optimal radial arterial waveform. In all, 10 radial arterial pressure waveforms were recorded. HEM-9010AI is a prototype of HEM-9000AI and requires the measurement of blood pressure in the upper arm. Therefore, the blood pressure in the upper arm was determined by HEM-907. Then, the first and second peaks of the peripheral systolic pressure (SBP1 and SBP2) and radial diastolic pressure (DBP) were automatically detected, and the radial AI was calculated as follows: (SBP2-DBP)/(SBP1-DBP) $\times 100(\%)$. Waveform data were confirmed by well-trained technicians, and subjects with a standard deviation of rAI recordings over $6 \%$ were excluded.

A good reproducibility of radial pressure wave reflection at longer time intervals (hours and weeks) has been demonstrated. ${ }^{18}$ In the present study, the reproducibility (i.e. hour-to-hour variability) was assessed in 38 subjects on the same day, but at different times. Pearson's correlation coefficients of AI between visit 1 and visit 2 were $0.95(P<0.01)$, and the coefficient of variation of rAI was $3.2 \%$. In these two visits, the coefficient of variation of systolic blood pressure was $4.0 \%$ and that of diastolic blood pressure was $4.2 \%$.

\section{Laboratory measurements}

Low-density lipoprotein (LDL) cholesterol, high-density lipoprotein (HDL) cholesterol, triglycerides (TG), fasting plasma glucose (FPG) and serum creatinine levels were measured using enzymatic methods (Falco Biosystems Co. Ltd, Tokyo, Japan). All the blood samples were obtained in the morning after the patients had fasted overnight.

\section{Statistical analysis}

Data were expressed as means \pm s.d. Univariate linear regression analysis was applied to assess the correlations of radial AI with other variables. Then, a stepwise multivariate linear regression analysis was conducted to identify the strength of the significance of the relationship of risk factors for CVD with AI. In addition, a general linear model (GLM) univariate linear regression analysis was applied to assess the independency and interaction of the correlations. The mean radial AI at each level of the age (increments of every 5 years) was compared with those at the previous level using a GLM univariate analysis. For the assessments of the differences in the status of each variable among the groups, a one-way analysis of variance (ANOVA) was applied. All the analyses were conducted using the SPSS software for Windows, version 11.0J (SPSS, Chicago, IL, USA); $P$-values of $<0.05$ were considered to denote statistically significant differences.

\section{RESULTS}

Of the 8940 subjects who agreed to participate in this study, 2202 were excluded because we failed to obtain a self report on smoking status $(n=75)$, failed to perform spirometry $(n=41)$, the standard deviation of the rAI was over $6 \%(n=354)$, FEV1.0\% was $<70 \%(n=241)$, the patient had a permanent pacemaker implantation or atrial fibrillation, or both $(n=69)$, or the patient was undergoing treatment for heart disease $(n=130)$, stroke $(n=77)$, renal disease $(n=87)$, pulmonary disease $(n=30)$, hypertension $(n=697)$, dyslipidemia $(n=281)$, diabetes mellitus $(n=85)$, collagen diseases $(n=12)$ and hormonal replacement therapy $(n=23)$. In addition, 144 subjects aged $<30$ years (most of them female) were excluded. Finally, 6594 subjects (3675 men and 2919 women) were included in the analysis. In this study,

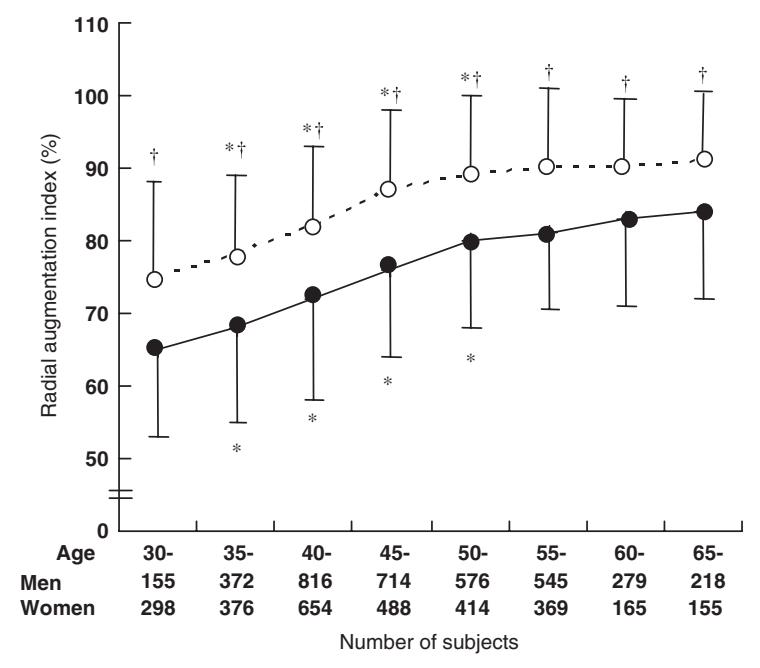

Figure 1 Relationship between 5-year age increments from $<30$ years of age to 65-84 years of age. Circles represent women; solid dots represent men; ${ }^{*} P<0.05$ vs. the value in the group with the previous level of mean blood pressure (as assessed by a general linear model multivariate analysis); $\dagger P<0.05$ vs. men.

Table 1 Results of general linear model univariate linear regression analysis to assess the independency of the effect of age, gender and their interaction on radial augmentation index

\begin{tabular}{lcrr}
\hline Variable & $B(95 \% \mathrm{Cl})$ & t-value & P-value \\
\hline Age & $0.30(0.26$ to 0.34$)$ & 15.02 & $<0.01$ \\
Gender & $-9.44(-11.98$ to -6.89$)$ & -7.28 & $<0.01$ \\
Interaction between & $0.07(0.02$ to 0.12$)$ & 2.94 & $<0.01$ \\
age and gender & & & \\
Height & $-0.40(-0.45$ to -0.36$)$ & -18.21 & $<0.01$ \\
BMI & $-0.55(-0.64$ to -0.46$)$ & -11.81 & $<0.01$ \\
Smoking & $4.57(4.00$ to 5.14$)$ & 15.74 & $<0.01$ \\
MBP & $0.30(0.28$ to 0.32$)$ & 27.72 & $<0.01$ \\
rPR & $-0.63(-0.65$ to 0.60$)$ & -48.92 & $<0.01$ \\
LDL & $0.01(-0.01$ to 0.01$)$ & 0.53 & 0.59 \\
HDL & $-0.06(-0.07$ to -0.03$)$ & -6.29 & $<0.01$ \\
TG & $0.01(0.00$ to 0.02$)$ & 1.98 & 0.05 \\
FPG & $0.01(-0.01$ to 0.03$)$ & 0.86 & 0.39 \\
\hline
\end{tabular}

Abbreviations: BMI, body mass index; 95\% CI, 95\% confidential interval; FPG, fasting plasma glucose; HDL, serum high-density lipoprotein cholesterol; LDL, serum low-density lipoprotein cholesterol; MBP, mean blood pressure in the right upper arm determined using the cholesterol; MBP, mean blood pressure in the right upper arm determined using the
oscillometric method; rPR, pulse rate determined using radial arterial pressure wave form oscillometric method; rPR, pulse rate determined using radial arterial pressure
analysis; Smoking, number of subjects with smoking; TG, serum triglycerides. $R^{2}=0.51$.

smoking status was self-reported and was graded into two categories (current smoking $=1$, former smoker or never smoking $=0$ ).

The subjects were classified based on their age in 5-year increments from 30 years of age to $65-84$ years of age in both genders. Figure 1 depicts the values of radial AI in each group. In both genders, although a phased increase of the radial AI with age could be confirmed until the age of 50 years, no further increase of the radial AI was observed with increase of age above 50 years. In all age categories, radial AI was higher in women than in men. A GLM univariate linear regression analysis showed that age and gender are independent variables related to $\mathrm{AI}$, and they had a significant interaction (Table 1). Then, the analyses were conducted in four groups classified by age and gender (that is, men $<50$ years of age, men $\geqslant 50$ years of age, women $<50$ years of age and women $\geqslant 50$ 
Table 2 Clinical characteristics in study subjects in four groups classified by age and gender

\begin{tabular}{|c|c|c|c|c|c|c|}
\hline & $M e n<50$ & $M e n \geqslant 50$ & Women $<50$ & Women $\geqslant 50$ & F-value & P-value \\
\hline Age (years) & $42 \pm 5$ & $58 \pm 6^{*}$ & $41 \pm 5^{* \dagger}$ & $58 \pm 6^{* \ddagger}$ & - & - \\
\hline BMI $\left(\mathrm{kg} \mathrm{m}^{-2}\right)$ & $23.6 \pm 3.0$ & $23.3 \pm 2.6^{*}$ & $20.8 \pm 3.0^{* \dagger}$ & $21.3 \pm 2.8^{* \dagger+}$ & 33.54 & $<0.01$ \\
\hline Smoking (\%) & $742(36)$ & $475(29)^{*}$ & $275(15)^{* \dagger}$ & $101(9) * * \neq$ & 0.12 & 0.73 \\
\hline rAl (\%) & $71.9 \pm 13.2$ & $81.5 \pm 11.6^{*}$ & $81.3 \pm 12.5^{*}$ & $90.0 \pm 10.8^{* \dagger \ddagger}$ & 1.89 & 0.17 \\
\hline rPR & $69 \pm 10$ & $67 \pm 10^{*}$ & $69 \pm 9^{\dagger}$ & $67 \pm 9 * \ddagger$ & 0.26 & 0.61 \\
\hline $\mathrm{SBP}(\mathrm{mm} \mathrm{Hg})$ & $118 \pm 14$ & $126 \pm 16^{*}$ & $107 \pm 13^{* \dagger}$ & $120 \pm 17^{\text {*怰 }}$ & 46.94 & $<0.01$ \\
\hline $\mathrm{DBP}(\mathrm{mm} \mathrm{Hg})$ & $73 \pm 11$ & $77 \pm 11^{*}$ & $65 \pm 10^{* \dagger}$ & 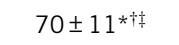 & 3.14 & 0.08 \\
\hline $\mathrm{MBP}(\mathrm{mm} \mathrm{Hg})$ & $88 \pm 12$ & $93 \pm 12^{*}$ & $79 \pm 11^{* \dagger}$ & $87 \pm 13^{* 中 \ddagger}$ & 13.95 & $<0.01$ \\
\hline $\operatorname{LDL}\left(\mathrm{mmol}^{-1}\right)$ & $3.3 \pm 0.9$ & $3.5 \pm 1.0^{*}$ & $2.9 \pm 0.7^{* \dagger}$ & $3.7 \pm 0.8^{*+\frac{1}{4}}$ & 200.19 & $<0.01$ \\
\hline $\mathrm{HDL}\left(\mathrm{mmol} \mathrm{I}^{-1}\right)$ & $1.5 \pm 0.4$ & $1.5 \pm 0.4$ & $1.9 \pm 0.4^{* \dagger}$ & $1.9 \pm 0.4^{* \dagger}$ & 3.32 & 0.07 \\
\hline
\end{tabular}

Abbreviations: $<50$, age under $50 ; \geqslant 50$, age 50 or over; BMI, body mass index; DBP, diastolic blood pressure in the right upper arm determined using an oscillometric method; $F$-value, $F$-value of the interaction between the effect of age and that of gender on variables; FPG, fasting plasma glucose; HDL, serum high-density lipoprotein cholesterol; LDL, serum low-density lipoprotein cholesterol; MBP, mean blood pressure in the right upper arm determined using an oscillometric method; $P$-value, $P$-value of the interaction between the effects of age and gender on variables; $\mathrm{rAI}$, radial augmentation index; rPR, pulse rate determined using radial arterial pressure wave form analysis; SBP, systolic blood pressure in the right upper arm determined using an oscillometric method; Smoking, number of subjects with smoking; SBP1, first peak of the radial systolic blood pressure; SBP2, second peak of the radial systolic blood pressure; TG, serum triglycerides. ${ }^{*} P<0.05$ vs. men aged $<50$ years.

$P<0.05$ vs. men aged $\geqslant 50$ years.

$\ddagger P<0.05$ vs. women aged $<50$ years.

Table 3 Results of univariate linear regression analysis conducted to assess the relationship of radial augmentation index to other clinical variables including the risk factors for cardiovascular disease in four groups classified by age and gender

\begin{tabular}{|c|c|c|c|c|c|c|c|c|}
\hline \multirow[b]{2}{*}{ Variable } & \multicolumn{2}{|c|}{$M e n<50$} & \multicolumn{2}{|c|}{$M e n \geqslant 50$} & \multicolumn{2}{|c|}{ Women $<50$} & \multicolumn{2}{|c|}{ Women $\geqslant 50$} \\
\hline & $r$ & e & $r$ & se & $r$ & se & $r$ & P-valu \\
\hline ge & 0.29 & $<0.01$ & .15 & $<0.01$ & 0.34 & D1 & 0.06 & 0.04 \\
\hline Heigl & -0.25 & $<0.01$ & -0.27 & 1 & -0.19 & & -0.18 & $<0.01$ \\
\hline BMI & 0.04 & & -0.10 & & U3 & 23 & -0.03 & 0.3 \\
\hline Smc & 0.21 & $<0$ & & 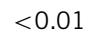 & 0.15 & 1 & 0.08 & $<0.0$ \\
\hline rPR & -0.37 & $<0.01$ & -0.46 & $<0.01$ & -0.34 & 01 & -0.49 & $<0.01$ \\
\hline $\mathrm{MBI}$ & 0.21 & $<0.0$ & & & & & & $<0.01$ \\
\hline LDL & & & -0 & & & 10 & -0 & 0.27 \\
\hline $\mathrm{HDL}$ & -0.01 & & -0.09 & $<0.01$ & -0.03 & 0.15 & -0.02 & 0.5 \\
\hline TG & 0.04 & 0.05 & -0.02 & 0.49 & 0.09 & $<0.01$ & -0.01 & 0.87 \\
\hline FPG & -0.01 & 0.62 & -0.04 & 0.16 & 0.04 & 0.09 & -0.06 & 0.05 \\
\hline
\end{tabular}

Abbreviations: $<50$, age under $50 ; \geqslant 50$, age 50 or over; BMI, body mass index; FPG, fasting plasma glucose; HDL, serum high-density lipoprotein cholesterol; LDL, serum low-density lipoprotein cholesterol; MBP, mean blood pressure in the right upper arm determined using an oscillometric method; rPR, pulse rate determined using radial arterial pressure wave form analysis; TG, serum triglycerides.

years of age). Table 2 depicts the clinical characteristics, including the risk factors for CVD (smoking status, body mass index, blood pressure, LDL, TG, HDL and FPG), in those four groups. ANOVA showed that the highest value of mean of AI was observed in women aged $\geqslant 50$ years.

In the following analyses, mean blood pressure was used as a covariate because it is a determinant of large arterial stiffness and peripheral reflectance of pressure wave reflection, and both were major determinants of AI. ${ }^{19,20}$

As shown in Table 3, univariate linear regression analysis showed that smoking and mean blood pressure had a significant correlation with AI in all of those four groups. A step-wise multivariate linear regression analysis conducted after adjustment of age, height and pulse rate showed that, among the risk factors for CVD, mean blood pressure has a close positive relationship with AI in all four groups, and that smoking also has a close positive relationship with AI, except in women aged $\geqslant 50$ years (Table 4 ).

A GLM univariate linear regression analysis showed that mean blood pressure and smoking are independent significant variables related to radial AI, with interaction in men of all ages. On the contrary, in women, mean blood pressure and smoking did not have a significant interaction (Table 5).

\section{DISCUSSION}

This cross-sectional study succeeded in measuring radial AI in a large number of healthy male and female subjects, aged from 30 to 82 years, and the results of this study were consistent with the results of an earlier study (that is, age-related increase in AI was prominent in subjects under 50 years of age, and AI was higher in women than in men). ${ }^{13}$ Thus, evaluation of the effects of age and gender on the relationships between risk factors for CVD and AI is essential. Several studies have shown that hypertension, abnormal glucose metabolism, adiposity, smoking and heart rate are related to $\mathrm{AI} .{ }^{7-11,21}$ In the present study, although blood pressure was an independent variable related to increased $\mathrm{AI}$ in men and women at any age, the significance of smoking related to increased AI was limited to men of any age and women aged $<50$ years. To the best of our knowledge, this study is the first to suggest the gender-related difference of the relationship between risk factors for CVD and AI even in healthy individuals.

Recently, McEniery et al. ${ }^{22}$ demonstrated that the risk factors for CVD were independently related to central blood pressure, which was estimated by AI via the generalized transfer function. However, no study has evaluated the effect of the cluster of risk factors for CVD on AI. In the present study, blood pressure and smoking were found to act independently to increase AI without any interaction in young 
Table 4 Results of a step-wise multivariate linear regression analysis to assess the strength of the relationships of risk factors for cardiovascular disease with radial augmentation index adjusted for age, height and pulse rate in four groups classified by age and gender

\begin{tabular}{|c|c|c|c|}
\hline Variable & $\beta$ & $\mathrm{t}$-value & P-value \\
\hline \multicolumn{4}{|c|}{ Men $<50\left(R^{2}=0.46\right)$} \\
\hline MBP & 0.33 & 17.72 & $<0.01$ \\
\hline Smoking & 0.20 & 11.97 & $<0.01$ \\
\hline BMI & -0.15 & -8.03 & $<0.01$ \\
\hline $\mathrm{HDL}$ & -0.06 & -3.28 & $<0.01$ \\
\hline TG & 0.04 & 2.34 & 0.02 \\
\hline LDL & - & - & - \\
\hline FPG & - & - & - \\
\hline \multicolumn{4}{|c|}{ Men $\geqslant 50\left(R^{2}=0.41\right)$} \\
\hline MBP & 0.24 & 11.77 & $<0.01$ \\
\hline Smoking & 0.18 & 9.26 & $<0.01$ \\
\hline BMI & -0.18 & -8.45 & $<0.01$ \\
\hline HDL & -0.13 & -6.27 & $<0.01$ \\
\hline LDL & - & - & - \\
\hline TG & - & - & - \\
\hline FPG & - & - & - \\
\hline \multicolumn{4}{|c|}{ Women $<50\left(R^{2}=0.41\right)$} \\
\hline MBP & 0.33 & 16.40 & $<0.01$ \\
\hline Smoking & 0.10 & 5.16 & $<0.01$ \\
\hline BMI & -0.13 & -6.41 & $<0.01$ \\
\hline $\mathrm{TG}$ & 0.06 & 2.98 & $<0.01$ \\
\hline HDL & -0.06 & -2.80 & $<0.01$ \\
\hline LDL & - & - & - \\
\hline FPG & - & - & - \\
\hline \multicolumn{4}{|c|}{ Women $\geqslant 50\left(R^{2}=0.36\right)$} \\
\hline MBP & 0.25 & 9.56 & $<0.01$ \\
\hline BMI & -0.12 & -4.33 & $<0.01$ \\
\hline HDL & -0.06 & -2.30 & 0.02 \\
\hline Smoking & - & - & - \\
\hline LDL & - & - & - \\
\hline TG & - & - & - \\
\hline FPG & - & - & - \\
\hline
\end{tabular}

Abbreviations: $<50$, age under $50 ; \geqslant 50$, age 50 or over; BMI, body mass index; FPG, fasting plasma glucose; HDL, serum high-density lipoprotein cholesterol; $L D L=$ serum low-density lipoprotein cholesterol; MBP, mean blood pressure in the right upper arm determined using an oscillometric method; TG, serum triglycerides.

women. On the contrary, in men, blood pressure and smoking had synergistic effects on the increase in AI even in the elderly. Thus, the present study suggested the clustering effect of smoking and blood pressure on the increase in AI and its gender difference. In subjects with hypertension, the exaggerated effect of smoking on the increase in AI might be more prominent in men than in women.

Blood pressure and smoking increased arterial stiffness through various mechanisms. ${ }^{1-3,23}$ Both factors increase oxidative stress, activate vascular inflammation and/or produce endothelial dysfunction, and these abnormalities increase arterial stiffness. ${ }^{1-3,23,24}$ Furthermore, neurohormonal abnormalities related to hypertension also increase arterial stiffness. ${ }^{23,24}$ Consistent with the results of Jatoi's study, the present study confirmed that smoking increases AI independent of blood pressure, ${ }^{11}$ and therefore the mechanisms of the increase in AI might be different, at least in part.
Table 5 Results of general linear model univariate linear regression analysis to assess the effect of interaction between smoking and mean blood pressure on radial augmentation index adjusted for age, height, pulse rate, body mass index, serum low-density lipoprotein cholesterol, serum high-density lipoprotein cholesterol, serum triglycerides and fasting plasma glucose in four groups classified by age and gender

\begin{tabular}{|c|c|c|c|}
\hline Variable & B $(95 \%$ Cl) & t-value & P-value \\
\hline \multicolumn{4}{|l|}{ Men $<50\left(R^{2}=0.44\right)$} \\
\hline Smoking & $11.62(5.00-18.23)$ & 3.44 & $<0.01$ \\
\hline Mean blood pressure & $0.38(0.34-0.43)$ & 15.82 & $<0.01$ \\
\hline $\begin{array}{l}\text { Interaction between smoking } \\
\text { and mean blood pressure }\end{array}$ & $0.07(0.01-0.12)$ & 1.91 & 0.04 \\
\hline \multicolumn{4}{|l|}{ Men $\geqslant 50\left(R^{2}=0.42\right)$} \\
\hline Smoking & $12.50(5.35-19.64)$ & 3.43 & $<0.01$ \\
\hline Mean blood pressure & $0.25(0.21-0.29)$ & 11.13 & $<0.01$ \\
\hline $\begin{array}{l}\text { Interaction between smoking } \\
\text { and mean blood pressure }\end{array}$ & $0.08(0.01-0.16)$ & 2.23 & 0.03 \\
\hline \multicolumn{4}{|l|}{ Women $<50\left(R^{2}=0.40\right)$} \\
\hline Smoking & $3.29(-5.89-12.47)$ & 0.70 & 0.48 \\
\hline Mean blood pressure & $0.37(0.32-0.42)$ & 15.21 & $<0.01$ \\
\hline $\begin{array}{l}\text { Interaction between smoking } \\
\text { and mean blood pressure }\end{array}$ & $-0.01(-0.12-0.12)$ & -0.03 & 0.97 \\
\hline \multicolumn{4}{|l|}{ Women $\geqslant 50\left(R^{2}=0.34\right)$} \\
\hline Smoking & $0.43(-12.04-12.90)$ & 0.07 & 0.95 \\
\hline Mean blood pressure & $0.21(0.17-0.26)$ & 9.14 & $<0.01$ \\
\hline $\begin{array}{l}\text { Interaction between smoking } \\
\text { and mean blood pressure }\end{array}$ & $0.02(-0.13-0.17)$ & 0.20 & 0.84 \\
\hline
\end{tabular}

Abbreviations: $<50$, age under $50 ; \geqslant 50$, age 50 or over; $95 \% \mathrm{Cl}, 95 \%$ confidential interval.

Matsui et al. ${ }^{15}$ reported the gender difference of the relationship between carotid AI and left ventricular hypertrophy in subjects with hypertension under anti-hypertensive medication. They proposed that there was no capacity for carotid AI to be influenced by other pathological factors in women because of its higher value. However, even in young women aged $<50$ years, the present study demonstrated gender difference of the effect of blood pressure and smoking on AI. Mitchell et al. ${ }^{25}$ suggested unexplained differences in arterial structure or function between men and women that lead to increased wave reflection in women, and the results of the present study might agree with this suggestion.

This study has the following limitations: (1) It was conducted in healthy subjects. AI is increased severely in subjects with $\mathrm{CVD},{ }^{1-3,23,24,26}$ and therefore it is necessary to evaluate the usefulness of AI as a marker of arterial stiffness in subjects with CVD. (2) Although medications for CVD risk factors affect $\mathrm{AI},{ }^{1-3,23,24,26}$ the present study did not examine the influence of these medications on the relationship between the CVD risk factors and AI. (3) A further study to examine the ethnical difference of the relationship between the CVD risk factors and AI is proposed. (4) Body mass index correlated negatively with AI in this study. Further study is necessary to examine the relationship of pressure wave reflection with obesity. (5) The number of subjects aged $>60$ years was small not only among men but also among women. Furthermore, the number of smokers among women aged $\geqslant 50$ years was also relatively small as compared with other groups, and therefore confirmation of the effect of smoking 
on AI in elderly women is necessary. (6) This study did not examine the effect of former smoking on AI. (7) In cases with a higher value of radial AI, a discrepancy with the value of aortic AI has been suggested. ${ }^{27}$ Therefore, this might be a limitation of automated measurements of radial AI.

\section{Conclusion}

Among the risk factors for CVD, smoking and blood pressure were independent factors related to increase in AI. While age-related attenuation of increase in AI was confirmed in Japanese healthy subjects, these risk factors may act to increase AI even in elderly subjects, at least in part. However, a gender difference of the effect of these factors on AI may be present, and these factors may act synergistically to increase AI in men. On the contrary, these factors may act independently to increase AI without interaction in young women, whereas only the blood pressure seems to act to increase AI in elderly women. Thus, the co-existence of smoking and the elevation of blood pressure may have a more detrimental effect on pressure wave reflection in men than in women.

\section{ACKNOWLEDGEMENTS}

We are indebted to Professor J Patrick Barron of the International Medical Communications Center of Tokyo Medical University for his review of this paper.

\section{CONFLICT OF INTEREST}

This study was conducted by a grant from Omron Healthcare Corporation. The equipment to measure radial augmentation index was rented from Omron Healthcare Corporation and Mutsuo Yamazaki. Yasuhiro Sagawa and Yoshinori Miyawaki are employees of Omron Healthcare Corporation.

1 Safar ME. Pulse pressure, arterial stiffness, and cardiovascular risk. Curr Opin Cardiol 2003; 15: 258-263.

2 O'Rourke MF, Hashimoto J. Mechanical factors in arterial aging: a clinical perspective. J Am Coll Cardiol 2007; 50: 1-13.

3 Laurent S, Boutouyrie P. Recent advances in arterial stiffness and wave reflection in human hypertension. Hypertension 2007; 49: 1202-1206.

4 Guerin AP, Pannier B, Marchais SJ, London GM. Cardiovascular disease in the dialysis population: prognostic significance of arterial disorders. Curr Opin Nephrol Hypertens 2006; 15: 105-110.

5 Williams B, Lacy PS, Thom SM, Cruickshank K, Stanton A, Collier D, Hughes AD, Thurston H, O'Rourke M, CAFE Investigators; Anglo-Scandinavian Cardiac Outcomes Trial Investigators; CAFE Steering Committee and Writing Committee. Differential impact of blood pressure-lowering drugs on central aortic pressure and clinical outcomes: principal results of the Conduit Artery Function Evaluation (CAFE) study. Circulation 2006; 113: 1213-1225.

6 Chirinos JA, Zambrano JP, Chakko S, Veerani A, Schob A, Willens HJ, Perez G, Mendez AJ. Aortic pressure augmentation predicts adverse cardiovascular events in patients with established coronary artery disease. Hypertension 2005; 45: 980-985.
7 Duprez DA, Kaiser DR, Whitwam W, Finkelstein S, Belalcazar A, Patterson R, Glasser S, Cohn JN. Determinants of radial artery pulse wave analysis in asymptomatic individuals. Am J Hypertens 2004; 17: 647-653.

8 Schram MT, Henry RM, van Dijk RA, Kostense PJ, Dekker JM, Nijpels G, Heine RJ, Bouter LM, Westerhof N, Stehouwer CD. Increased central artery stiffness in impaired glucose metabolism and type 2 diabetes: the Hoorn Study. Hypertension 2004; 43: $176-181$.

9 Vergnaud AC, Protogerou AD, Li Y, Czernichow S, Vesin C, Blacher J, Safar ME. Pulse pressure amplification, adiposity and metabolic syndrome in subjects under chronic antihypertensive therapy: the role of heart rate. Atherosclerosis 2007; 199: 222-229; e-pub ahead of print.

10 Aznaouridis K, Vlachopoulos C, Dima I, loakeimidis N, Stefanadis C. Triglyceride level is associated with wave reflections and arterial stiffness in apparently healthy middleaged men. Heart 2007; 93: 613-614.

11 Jatoi NA, Jerrard-Dunne P, Feely J, Mahmud A. Impact of smoking and smoking cessation on arterial stiffness and aortic wave reflection in hypertension. Hypertension 2007; 49: 981-985.

12 McEniery CM, Yasmin, Hall IR, Qasem A, Wilkinson IB, Cockcroft JR, ACCT Investigators. Normal vascular aging: differential effects on wave reflection and aortic pulse wave velocity: the Anglo-Cardiff Collaborative Trial (ACCT). J Am Coll Cardiol 2005; 46: 1753-1760.

13 Dart AM, Gatzka CD, Kingwell BA, Willson K, Cameron JD, Liang YL, Berry KL, Wing LM, Reid CM, Ryan P, Beilin LJ, Jennings GL, Johnston CI, McNeil JJ, Macdonald GJ, Morgan TO, West MJ. Brachial blood pressure but not carotid arterial waveforms predict cardiovascular events in elderly female hypertensives. Hypertension 2006; 47: 785-790.

14 Matsui Y, Ishikawa J, Eguchi K, Hoshide S, Miyashita H, Shimada K, Kario K. The influence of wave reflection on left ventricular hypertrophy in hypertensives patients is modified by age and gender. Hypertens Res 2008; 31: 649-656.

15 Lees RS. Prevention of atherosclerosis progression in asymptomatic healthy elderly. Am J Clin Nutr 2007; 86: 1569S-1571S.

16 Takazawa K, Kobayashi H, Shindo N, Tanaka N, Yamashina A. Relationship between radial and central arterial pulse wave and evaluation of central aortic pressure using the radial arterial pulse wave. Hypertens Res 2007; 30: 219-228.

17 Melenovsky V, Borlaug BA, Fetics B, Kessler K, Shively L, Kass DA. Estimation of central pressure augmentation using automated radial artery tonometry. $J$ Hypertens 2007; 25: 1403-1409.

18 Papaioannou TG, Karatzis EN, Karatzi KN, Gialafos EJ, Protogerou AD, StamatelopouIos KS, Papamichael CM, Lekakis JP, Stefanadis Cl. Hour-to-hour and week-to-week variability and reproducibility of wave reflection indices derived by aortic pulse wave analysis: implications for studies with repeated measurements. J Hypertens 2007; 25: 1678-1686.

19 Stewart AD, Jiang B, Millasseau SC, Ritter JM, Chowienczyk PJ. Acute reduction of blood pressure by nitroglycerin does not normalize large artery stiffness in essential hypertension. Hypertension 2006; 48: 404-410.

20 Safar ME, Blacher J, Protogerou A, Achimastos A. Arterial stiffness and central hemodynamics in treated hypertensive subjects according to brachial blood pressure classification. J Hypertens 2008; 26: 130-137.

21 Wilkinson IB, Mohammad NH, Tyrrell S, Hall IR, Webb DJ, Paul VE, Levy T, Cockcroft JR. Heart rate dependency of pulse pressure amplification and arterial stiffness. Am J Hypertens 2002; 15 (1 Pt 1): 24-30.

22 McEniery CM, Yasmin, McDonnell B, Munnery M, Wallace SM, Rowe CV, Cockcroft JR, Wilkinson IB, Anglo-Cardiff Collaborative Trial Investigators. Central pressure: variability and impact of cardiovascular risk factors: the Anglo-Cardiff Collaborative Trial II. Hypertension 2008; 51: 1476-1482.

23 Zieman SJ, Melenovsky V, Kass DA. Mechanisms, pathophysiology, and therapy of arterial stiffness. Arterioscler Thromb Vasc Biol 2005; 25: 932-943.

24 Safar ME, Levy BI, Struijker-Boudier H. Current perspectives on arterial stiffness and pulse pressure in hypertension and cardiovascular diseases. Circulation 2003; 107: 2864-2869.

25 Mitchell GF, Parise H, Benjamin EJ, Larson MG, Keyes MJ, Vita JA, Vasan RS, Levy D. Changes in arterial stiffness and wave reflection with advancing age in healthy men and women: the Framingham Heart Study. Hypertension 2004; 43: 1239-1245.

26 Nichols WW. Clinical measurement of arterial stiffness obtained from noninvasive pressure waveforms. Am J Hypertens 2005; 18 (1 Pt 2): 3S-10S.

27 Millasseau SC, Patel SJ, Redwood SR, Ritter JM, Chowienczyk PJ. Pressure wave reflection assessed from the peripheral pulse: is a transfer function necessary? Hypertension 2003; 41: 1016-1020. 